\title{
ANALYSIS OF REJECTION CHARACTERISTICS OF MACROMOLECULAR GEL LAYER FOR LOW MOLECULAR WEIGHT SOLUTES IN ULTRAFILTRATION
}

\author{
SHIN-ICHI NAKAO, SATOSHI YUMOTO AND SHOJI KIMURA \\ Institute of Industrial Science, University of Tokyo, Tokyo 106
}

\begin{abstract}
Rejection characteristics of macromolecular gel layer for low-molecular weight solutes were studied by ultrafiltration using ovalbumin and polyvinyl alcohol, which formed a gel layer, and vitamin $\mathbf{B}_{12}$, raffinose and glucose, which were rejected by the gel layer. The bilayer membrane model and the Jagur-Grodzinski and Kedem equations were found effective in analyzing the rejection ability of the gel layer, which was defined by two parameters, $\sigma_{g}$ and $\boldsymbol{P}_{g}$. These parameters could be determined by the curve-fitting method. Furthermore, $\sigma_{g}$ and $\boldsymbol{P}_{\theta}$ were analyzed by use of the steric hindrance-pore model, and the structure of the gel layer was estimated. It became clear from the experimental results that compaction of the gel layer by mechanical pressure occurred and affected the rejection ability. This effect can be calculated by taking into the pressure dependencies of thickness and pore radius of gel layer.
\end{abstract}

\section{Introduction}

In membrane separation processes, it is well known that a gel layer of macromolecular substances or suspended matter tends to form on a membrane surface, and that it reduces the permeate flux and affects the rejection nature of membranes for lowmolecular weight solutes. Thus, it is very important for practical application to understand the nature of this gel layer.

We studied this problem in fouling experiments in which sewage and industrial water were treated by reverse osmosis, and the salt rejection ability of the deposit layer formed on a membrane surface was observed ${ }^{5}$. Sheppard et al. $^{11}$ ) have also reported the existence of a fouling layer which had the same ability. After this study, we next considered the characteristics of the macromolecular gel layer formed on an ultrafiltration membrane, and found it possible to analyze quantitatively the resistance of this layer to permeation ${ }^{9}$. As the last stage of out study of this problem, the analysis of rejection characteristics of the gel layer is necessary. This is the purpose of the present study.

The salt rejection ability of a gel layer of polyelectrolyte has already been utilized in a well-known separation method using a dynamically formed membrane, which was quantitatively regarded as a thin

\footnotetext{
Received March 3, 1982. Correspondence concerning this article should be addressed to S. Kimura. S. Yumoto is now with Nitto Electric Industrial Co., Ltd., Shimohozumi, Ibarakishi, Osaka 567.
}

ion exchange membrane ${ }^{12}$. However, Nomura and Kimura $^{10)}$ recently reported that this membrane could also reject low-molecular weight nonelectrolytes, sucrose, glucose and urea, as well as electrolytes. During hemofiltration in an artificial kidney, blood components as well as proteins form the gel layer, and Dorson et al. ${ }^{2)}$ have studied the rejection ability of this layer using model systems.

We have also reported the rejection ability of a protein gel layer for vitamin $B_{12}$ in ultrafiltration ${ }^{6}$. In the present study, we performed further experiments using two kinds of gel-forming solutes and three low-molecular weight solutes, and found it possible to analyze the rejection ability of gel layer by quantitatively applying the bilayer membrane model and pore model.

\section{Theoretical}

\subsection{Resistance of gel layer}

To analyze the rejection ability of the gel layer it is necessary to specify the state of the gel layer. The only index for this is the gel layer resistance to permeation flow, $R_{g}$, which was studied in our previous work ${ }^{9}$. According to the resistance law, this is defined as

$$
J_{v}=\frac{\Delta P}{R_{m}+R_{g}}=\frac{\Delta P_{m}}{R_{m}}=\frac{\Delta P_{g}}{R_{g}}
$$

where $R_{m}$ is resistance of a membrane. $\Delta P_{m}$ and $\Delta P_{g}$ are pressure differences acting on membrane and gel layer respectively, and are related to $\Delta P$ as follows. 


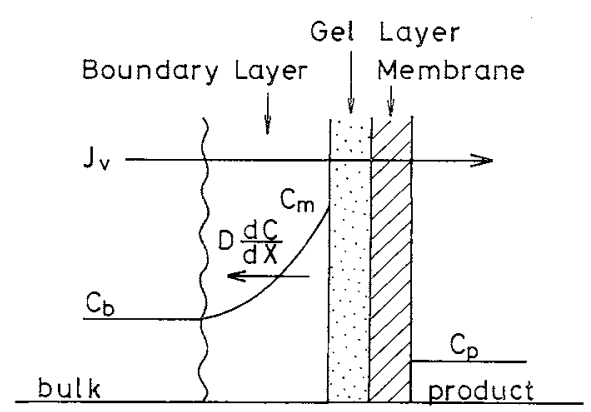

Fig. 1 Bilayer membrane model, schematic

$$
\Delta P=\Delta P_{m}+\Delta P_{g}
$$

\subsection{Rejection ability of gel layer}

In ultrafiltration, we have already made it clear that the rejection ability of a membrane is expressed by two membrane parameters: solute permeability $P$ and reflection coefficient $\sigma^{7}$. Thus, if one can determine these parameters of a gel layer, it is possible to analyze its rejection ability in the same manner.

For this analysis, transport equations for water and solute flow through two different membranes in series are necessary, and have been developed by Jagur-Grodzinski and Kedem ${ }^{4}$. Following the bilayer membrane model illustrated in Fig. 1, real rejection of these series membranes is given by

$$
\begin{aligned}
& R= \\
& \frac{\left(1-F_{g}\right)\left(1-\sigma_{m}\right)+F_{g}\left(1-\sigma_{g}\right)\left(1-F_{m} \sigma_{m}\right)-\left(1-\sigma_{g}\right)\left(1-\sigma_{m}\right)}{\left(1-F_{g}\right)\left(1-\sigma_{m}\right)+\bar{F}_{g}\left(1-\bar{\delta}_{g}\right)\left(1-F_{m} \sigma_{m}\right)}
\end{aligned}
$$

where

$$
\begin{aligned}
& F_{g}=\exp \left\{-\frac{J_{v}\left(1-\sigma_{g}\right)}{P_{g}}\right\} \\
& F_{m}=\exp \left\{-\frac{J_{v}\left(1-\sigma_{m}\right)}{P_{m}}\right\}
\end{aligned}
$$

In the case of a membrane with gel layer, suffix $g$ corresponds to gel layer and $m$ corresponds to membrane. In the case of known $\sigma_{m}$ and $P_{m}$, the values of $\sigma_{g}$ and $P_{g}$ are obtainable from experimental data by the curve-fitting method explained in our previous study ${ }^{7)}$.

Real rejection, $R$, in Eq. (2) is calculated by using the concentration polarization model, and mass transfer coefficient is needed in the calculation. This coefficient is usually determined by the velocity variation method $^{7}$. But it cannot be used under gel layer-forming conditions, because the gel layer thickness changes with velocity. Thus, the correlation which gives the mass transfer coefficient must be used to estimate its value.

In mixed solutions of gel layer-forming solute and low-molecular weight solute, it is not certain whether it is possible to use diffusivities in the aqueous solu- tion for the low-molecular weight solute. Regarding this problem, Hoshino and $\mathrm{Sato}^{3)}$ reported the diffusion of a small molecule in a polymer solution. They concluded that the diffusivity of a small solute was not affected by viscosity of mixed solution and was equal to the value in the single-solute aqueous solution.

Following this result, the mass transfer coefficient can be estimated by Deissler's equation ${ }^{7}$, using diffusivity in an aqueous solution and viscosity of a polymer solution.

\section{3 Steric hindrance-pore model}

The well-known pore model ${ }^{1,18)}$ is very useful in considering the physical meanings of $\sigma$ and $P$ and the membrane structure. In our previous work ${ }^{8)}$, we removed the wall correction factors from this model, and proposed the steric hindrance-pore model, In the present study, we attempted to apply this model to the macromolecular gel layer.

According to the steric hindrance-pore model, parameters expressing rejection ability, $\sigma$ and $P$, are defined by

$$
\begin{aligned}
& \sigma=1-S_{F}\left\{1+(16 / 9) q^{2}\right\} \\
& P=D \cdot S_{D} \cdot\left(A_{k} / \Delta X\right)
\end{aligned}
$$

where $q$ is the ratio of solute radius to pore radius, $A_{16}$ the ratio of total cross-sectional pore area to effective membrane area, and $\Delta X$ membrane thickness. $S_{D}$ and $S_{F}$ are the steric hindrance factors for diffusion and filtration flow respectively, and are given as

$$
\begin{aligned}
& S_{D}=(1-q)^{2} \\
& S_{F}=2(1-q)^{2}-(1-q)^{4}
\end{aligned}
$$

Applying Eqs. (5)-(8) to the gel layer, its structure can be characterized with $r_{p}$ and $A_{k} / \Delta X$.

The value of $A_{k} / \Delta X$ is also determined by pure water flux $J_{w}$ using the Hagen-Poiseuille equation as

$$
J_{w}=\left(r_{p}^{2} / 8 \mu\right) \cdot\left(A_{k} / \Delta X\right) \cdot \Delta P
$$

If the value of $r_{g}$ is known, $A_{k} / \Delta X$ can be calculated. 1. 4 Relationship among $P_{g}, A_{k} / \Delta X$ and $R_{g}$

We now apply Eq. (9) to the gel layer. In ultrafiltration, as the osmotic pressure of feed solution can be usually neglected, one can assume that solution flux $J_{v}$ in Eq. (1) is equal to pure water flux $J_{w}$ in Eq. (9). Therefore,

$$
\left(r_{p}^{2} / 8 \mu\right)\left(A_{k} / \Delta X\right)=1 / R_{g}
$$

Solute permeability of gel layer, $P_{g}$, is proportional to $A_{k} / \Delta X$ as shown in Eq. (6). Thus, the relationship among the three Parameters can be described as

$$
P_{g} \propto A_{k} / \Delta X \propto R_{g}^{-1}
$$

It is very interesting to learn whether this relationship can be verified by experimental data. 


\section{Experimental}

\section{1 Apparatus and materials}

The experimental apparatus used for ultrafiltration is the same as explained in our previous study ${ }^{9}$. Cellulose acetate tubular membranes, T2/A (nominal rejection of PEG $\left.\left(M_{w}, 6,000\right)=95.0-98.5 \%\right), \mathrm{T} 4 / \mathrm{A}$ $(50.0-80.0 \%)$ and T5/A (70.0-90.0\% for PEG $\left(M_{w}\right.$ 20,000)), were employed. All these membranes were supplied by Paterson Candy International, Ltd. England.

Two kinds of macromolecular solutes which form a gel layer on a membrane surface were used: the spherical polymer ovalbumin $\left(M_{w}=45,000\right)$ and the linear-chain polymer polyvinyl alcohol PVA224 $\left(M_{w}=100,000\right)$ supplied by Kurare Company, Japan.

As the low-molecular weight solutes which were rejected by a gel layer, three kinds of solutes were employed: vitamin $\mathrm{B}_{12}\left(M_{w}=1,355\right)$, raffinose $\left(M_{w}=\right.$ $504)$ and glucose $\left(M_{w}=180\right)$. Diffusivities of these solutes are $3.3,4.2$ and $6.9 \times 10^{-6} \mathrm{~cm}^{2} / \mathrm{s}^{7}$, and solute radii calculated by the Stokes-Einstein equation are $7.4,5.8$ and $3.6 \times 10^{-8} \mathrm{~cm}^{7}$, respectively.

\section{2 Conditions and procedure}

For the determination of rejection ability of gel layer, $\sigma_{g}$ and $P_{g}$, it is necessary to measure rejections at several points of different volume flux under the condition that only the index to specify the state of gel layer, $R_{y}$, should be kept constant. To give different flux, experiments must be performed at different pressures. But when bulk concentration is kept constant, the value of $R_{g}$ changes with pressure. Therefore, to keep $R_{g}$ constant it is necessary to change bulk concentration or feed velocity.

In these experiments, membrane parameters $\sigma_{m}$ and $P_{m}$ for vitamin $\mathrm{B}_{12}$, raffinose and glucose were first determined from the data of single-solute experiments by the method explained in our previous work $^{7}$. Then, experiments with mixed solutions were carried out at constant feed velocity, $58 \mathrm{~cm} / \mathrm{s}$, changing the concentration of ovalbumin or PVA224 to keep $R_{g}$ constant. These experiments were performed at three pressures: $4.5,6$ and $8 \times 10^{5} \mathrm{~Pa}$. However, it was very difficult to keep $R_{g}$ precisely constant. Finally, the experimental relationships between $R_{g}$ and real rejection, $R$, were obtained by graphing the data, and $R$ values were read at appropriate $R_{g}$ by interpolation. From these results, several sets of data, $R$ and $J_{v}$, were obtained for each value of $R_{g}$, and these data were then used to determine the parameters $\sigma_{g}$ and $P_{g}$ by the curvefitting method.

The temperature of feed solution was controlled at $25^{\circ} \mathrm{C}$ by a thermostat. The concentration of vitamin $B_{12}$ ranged from 50 to $100 \mathrm{ppm}$ and that of raffinose or glucose was 2,000 ppm.

The concentration of vitamin $\mathrm{B}_{12}$ was measured spectrophotometrically at $361 \mathrm{~nm}$ and concentrations of the other two solutes were analyzed by the phenolsulfuric acid method.

\section{Results and Discussion}

\section{1 Effects of a gel layer on solute rejection}

Before the quantitative analysis of solute rejection ability of a gel layer, changes of rejection due to the gel layer formation were examined by experiments of ultrafiltration with mixed ovalbumin-vitamin $B_{12}$ solutions. The term "rejection" here means the rejection of low-molecular weight solute.

Effects of flow rate and pressure are shown in Figs. 2 and 3 , respectively, for vitamin $B_{12}$ only and for the case with ovalbumin gel layer. It is clear from the results of flux changes with flow rate or pressure that membrane permeation is completely controlled by the gel layer. It is also apparent that observed rejections increase considerably due to the formation of ovalbumin gel layer. Increase of real rejection, which is determined after the correction of the effect of concentration polarization, is also observed in this case $\mathrm{e}^{6)}$.

\section{2 Determination of rejection ability}

Values of $\sigma_{m}$ and $P_{m}$ of the membranes used in experiments of mixed ovalbumin solution were respectively 0.89 and $1.1 \times 10^{-3} \mathrm{~cm} / \mathrm{s}$ for vitamin $B_{12}$, and 0.14 and $1.6 \times 10^{-3} \mathrm{~cm} / \mathrm{s}$ for raffinose. Glucose was not rejected by the membrane itself, so that the value of $\sigma_{m}$ was regarded as zero. For the membranes used in mixed PVA224 solution experiments, values of $\sigma_{m}$ and $P_{m}$ were 0.16 and $1.6 \times 10^{-3} \mathrm{~cm} / \mathrm{s}$ respectively.

The experimental relationships between $R_{g}$ and $R$ for mixed ovalbumin-raffinose solution are shown in Fig. 4. Assuming linear relationships, $R$ values were read at three $R_{g}$ values between 1,000 and 4,000 $\mathrm{MPa} \cdot \mathrm{s} / \mathrm{cm}$, and then used for the curve-fitting.

The plots of $R$ against $1 / J_{v}$ are shown in Figs. 5 and 6 for ovalbumin and PVA224 gel layer, respectively, and solid lines indicate Eq. (2) at each $R_{g}$ value. As illustrated in these figures, this equation fits the experimental data well except for less-rejected. solutions, glucose in Fig. 5 and vitamin $B_{12}$ in Fig. 6. (The reason for this lack of fit is discussed later.) It is obvious that Eq. (2) is appropriate for use as the transport equation for solute permeation through a gel layer.

Values of $\sigma_{g}$ and $P_{g}$ determined by the curve-fitting method are listed in Table 1. $\sigma_{g}$ of ovalbumin gel layer for raffinose and that of PVA224 gel layer ranges from 0.74 to 0.78 and from 0.53 to 0.61 with the change of $R_{g}$, so the values in Table 1 are average 

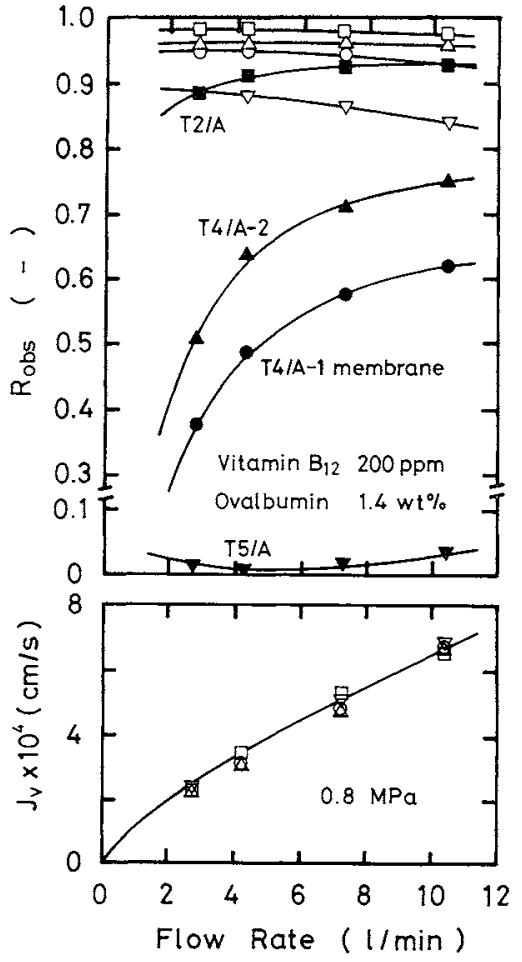

Fig. 2 Effects of flow rate on flux and observed rejection, using four membrances (black: vitamin $B_{12}$ single solute, white: mixed ovalbumin-vitamin $B_{12}$ solution)

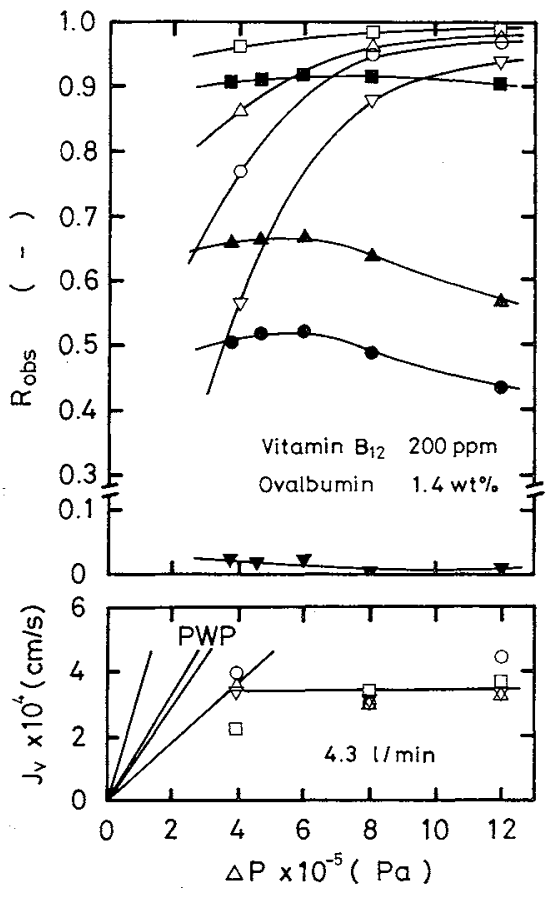

Fig. 3 Effects of pressure on flux and observed rejection (Keys are the same as in Fig. 2.)

ones. The relationships between $P_{g}$ and $R_{g}$ are shown in Fig. 7, and it is clear that $P_{g}$ is indeed inversely proportional to $R_{g}$ as estimated by Eq. (11).

Substituting values of $\sigma_{g}$ and $P_{g}$ listed in Table 1 into Eq. (2), real rejections can be calculated and are

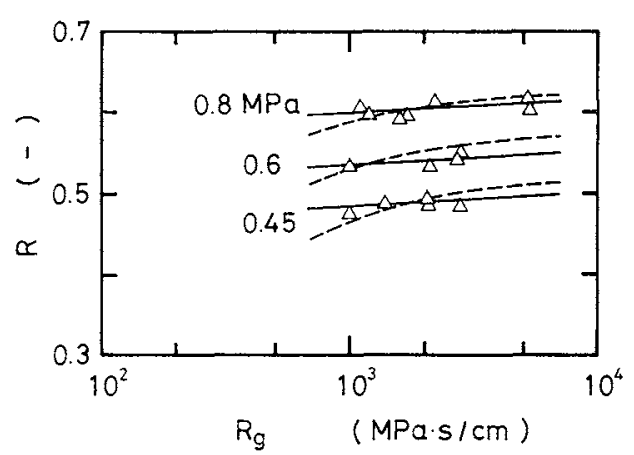

Fig. 4 Relationships between $R$ and $R_{g}$ for mixed ovalbumin-raffinose solution (dotted lines are calculated ones.)

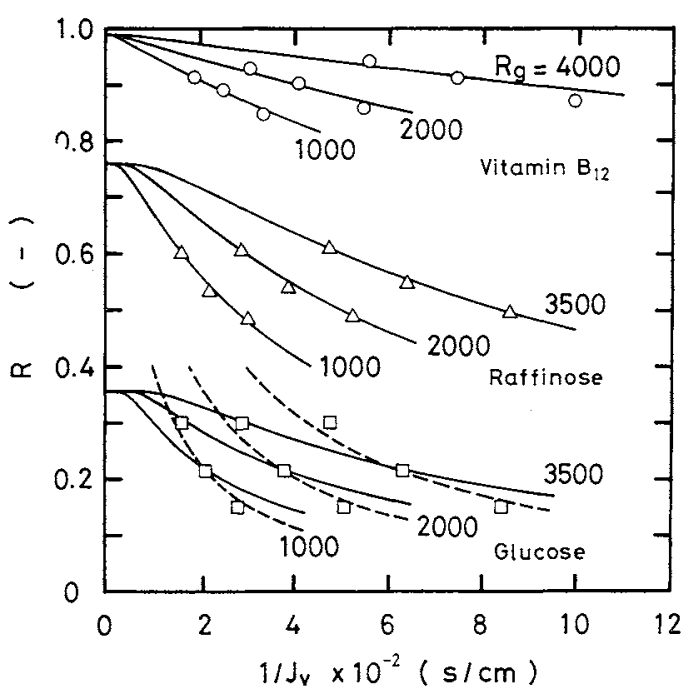

Fig. 5 Plots of $R$ vs. $1 / J_{v}$ in the case with ovalbumin gel layer (solid lines show values calculated by Eqs. (2), (3) and (4), dotted lines show values corrected for effect of compaction of gel layer.)

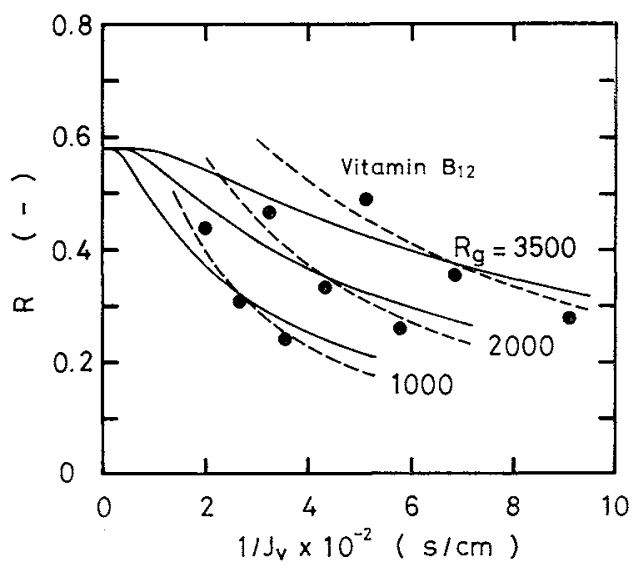

Fig. 6 Plots of $R$ vs. $1 / J_{v}$ in the case with PVA224 gel layer (Solid and dotted lines have same meaning as in Fig. 5.)

plotted against $R_{g}$ in Fig. 4 with dotted lines. Relationships between $R$ and $R_{g}$ are not linear, but within the range of $R_{g}$ employed in this study, $1,000-4,000$ $\mathrm{MPa} \cdot \mathrm{s} / \mathrm{cm}$, the calculated lines almost agree with the linear ones. Therefore, $R$ values read by interpola- 


\begin{tabular}{|c|c|c|c|c|c|}
\hline \multirow{2}{*}{ Solute } & \multicolumn{5}{|c|}{1 Values of $\sigma_{g}, P_{g}, r_{p}$ and $A_{k} / \Delta X$} \\
\hline & $\begin{array}{c}R_{g} \\
{[\mathrm{MPa} .} \\
\mathrm{s} / \mathrm{cm}]\end{array}$ & {$[-]$} & $\begin{array}{c}P_{g} \times 10^{4} \\
{[\mathrm{~cm} / \mathrm{s}]}\end{array}$ & $\begin{array}{c}r_{p} \times 10^{8} \\
{[\mathrm{~cm}]}\end{array}$ & $\begin{array}{l}A_{k} / \Delta X \\
{\left[\mathrm{~cm}^{-1}\right]}\end{array}$ \\
\hline \multicolumn{6}{|c|}{ Ovalbumin gel layer } \\
\hline \multirow[t]{3}{*}{ Glucose } & 1000 & 0.36 & 4.4 & 7.4 & 245 \\
\hline & 2000 & & 2.5 & & 139 \\
\hline & 3500 & & 1.5 & & 84 \\
\hline \multirow[t]{3}{*}{ Raffinose } & 1000 & 0.76 & 2.2 & 7.7 & 775 \\
\hline & 2000 & & 1.3 & & 495 \\
\hline & 3500 & & 0.82 & & 339 \\
\hline \multirow[t]{3}{*}{ Vitamin $\mathbf{B}_{12}$} & 1000 & 0.99 & 0.51 & 7.7 & 9660 \\
\hline & 2000 & & 0.27 & & 5110 \\
\hline & 4000 & & 0.12 & & 2270 \\
\hline \multicolumn{6}{|c|}{ PVA224 gel layer } \\
\hline \multirow[t]{3}{*}{ Vitamin $\mathrm{B}_{12}$} & 1000 & 0.58 & 3.4 & 11.6 & 677 \\
\hline & 2000 & & 2.0 & & 468 \\
\hline & 3500 & & 1.2 & & 315 \\
\hline
\end{tabular}

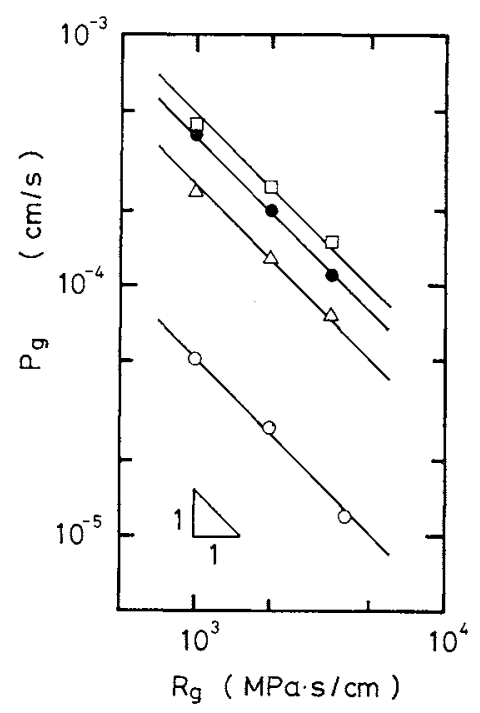

Fig. 7 Relationships between $\boldsymbol{P}_{g}$ and $\boldsymbol{R}_{g}$ (Keys are the same as in Figs. 5 and 6.)

tion of the linear line are valid.

\section{3 Application of the steric hindrance-pore model}

According to the analysis based on the steric hindrance-pore model, pore radius of gel layer, $r_{p}$, is first calculated by Eq. (5), and the results are given in Table 1. The $r_{p}$ values of ovalbumin gel layer were in good agreement and the average value $7.6 \times$ $10^{-8} \mathrm{~cm}$ was obtained. Pore radius of PVA224 gel layer was $11.6 \times 10^{-8} \mathrm{~cm}$.

Next, the value of $A_{k} / \Delta X$ can be determined by Eq. (6) with $r_{p}$ calculated above, and the results are also listed in Table 1. In the case of ovalbumin gel layer, values for vitamin $B_{12}$ are much larger than those for glucose and raffinose. $\sigma_{g}$ for vitamin $B_{12}$ is very close to unity, as shown in Table 1 , so the steric hindrance-pore model cannot be applied to this system. The relationships between $A_{k} / \Delta X$ and $R_{g}$ are plotted in Fig. 8. It is clear that $A_{k} / \Delta X$ is inversely proportional to $R_{g}$ as shown by Eq. (11).

$A_{k} / \Delta X$ can be also given by Eq. (9) with $r_{p}$ obtained,

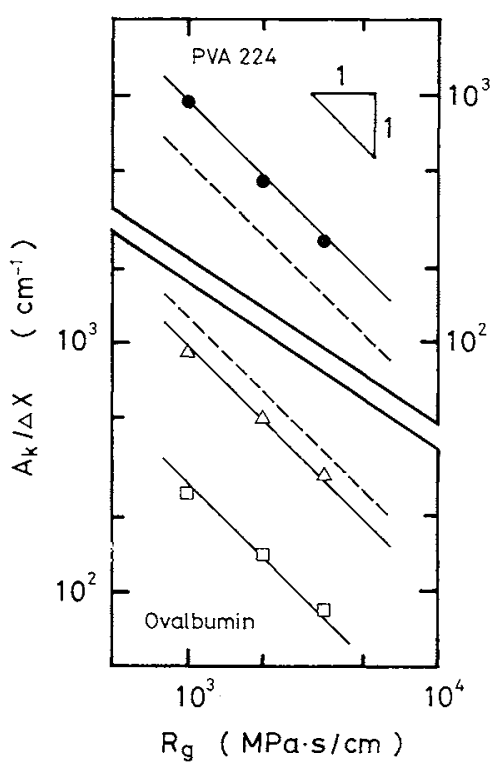

Fig. 8 Relationships between $A_{k} / \Delta X$ and $R_{g}$ (Dotted lines show the values calculated by Eq. (9). Keys are the same as in Figs. 5 and 6.)

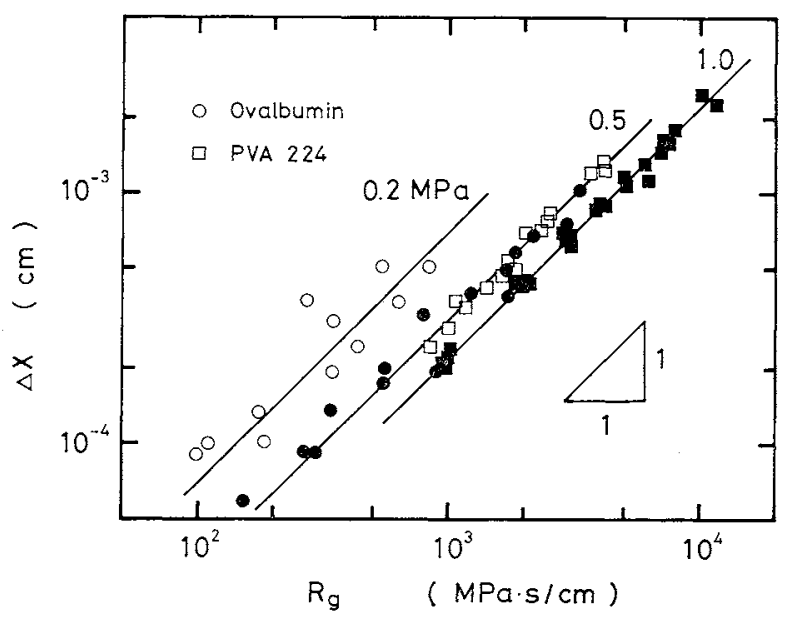

Fig. 9 Thickness of gel layer measured and its pressure dependency

and results are shown in Fig. 8 with dotted lines. The values calculated by Eq. (6) and those by Eq. (9) agree well. There is a little disagreement between Eq. (9) and values for glucose; the reason is not clear at this stage.

\section{4 Effect of compaction on rejection ability}

As $A_{k}$ is constant for a certain gel layer, Eq. (11) means that the thickness of gel layer, $\Delta X$, is proportional to $R_{g}$. In our previous study ${ }^{9}$, we weighed the gel layer formed on a membrane surface. The density of the gel layer is almost 1.0 , and $\Delta X$ can be calculated from this weight. As illustrated in Fig. 9, $\Delta X$ is indeed proportional to $R_{g}$ in both ovalbumin and PVA224 gel layer.

However, Fig. 9 also shows that $\Delta X$ depends on pressure at the point of the same $R_{g}$. This is the 
compaction of gel layer, and it affects rejection ability. If the pressure dependence of $\Delta X$ can be defined as an exponential function, the following relationship is obtained from Fig. 9.

$$
\Delta X \propto R_{g} \cdot \Delta P^{-\alpha}
$$

The exponent $\alpha$ is 0.89 for ovalbumin gel layer and 0.45 for PVA224 gel layer. The effect of this compaction by mechanical pressure is the reason of the disagreement in Figs. 5 and 6 mentioned before. Then this compaction effect on solute rejection is calculated as follows.

As $A_{k}$ is proportional to $r_{p}^{2}$, using Eq. (10) and (12), the following relationships are obtained in the case of constant $R_{g}$.

$$
\begin{aligned}
& r_{p} \propto \Delta P^{-\alpha / 4} \\
& A_{k} / \Delta X \propto \Delta P^{\alpha / 2}
\end{aligned}
$$

Thus, substituting Eqs. (13) and (14) into Eqs. (1) and (6), the effect of compaction on real rejection $R$ can be calculated by using Eq. (2). The results are shown in Figs. 5 and 6 with dotted lines. It is obvious from these figures that correction for effect of compaction of gel layer can be calculated by Eqs. (13) and (14).

\section{Conclusion}

In this study, rejection characteristics of macromolecular gel layer for low-molecular weight solutes in ultrafiltration were analyzed, and the following results were obtained.

Equation (2) based on the bilayer membrane model is applicable as the transport equation of solute permeation through gel layer, and the parameters expressing rejection ability of gel layer, $\sigma_{g}$ and $P_{g}$, are obtained by the curve-fitting method.

The physical meanings of $\sigma_{g}$ and $P_{g}$ and the structure of gel layer can be explained by the steric hindrance-pore model. According to this model, $P_{g}$ and $A_{k} / \Delta X$ are inversely proportional to $R_{g}$, and this relationship was verified by the experiments.

It became clear from the experimental results that compaction of gel layer by mechanical pressure affected the rejection ability. Furthermore, this effect can be calculated by using the pressure dependencies

\begin{tabular}{|c|c|c|c|}
\hline$A_{k}$ & & $\begin{array}{l}\text { ratio of total cross-sectional pore are } \\
\text { effective membrane area }\end{array}$ & ea to \\
\hline$D$ & $=$ & diffusivity & {$\left[\mathrm{cm}^{2} / \mathrm{s}\right]$} \\
\hline$F_{g}, F_{m}$ & $=$ & $\begin{array}{l}\text { quantities defined by Eqs. (3) and (4) } \\
\text { respectively }\end{array}$ & {$[-]$} \\
\hline$J_{v}$ & $=$ & volume flux through a membrane & {$\left[\mathrm{cm}^{3} / \mathrm{cm}^{2} \cdot \mathrm{s}\right]$} \\
\hline$J_{w}$ & $=$ & volume flux of pure water & {$\left[\mathrm{cm}^{3} / \mathrm{cm}^{2} \cdot \mathrm{s}\right]$} \\
\hline$P$ & $=$ & solute permeability & {$[\mathrm{cm} / \mathrm{s}]$} \\
\hline$\Delta P$ & $=$ & pressure difference & {$[\mathrm{Pa}]$} \\
\hline$q$ & $=$ & ratio of solute radius to pore radius & {$[-]$} \\
\hline$R$ & $=$ & real rejection & {$[-]$} \\
\hline$R_{g}, R_{m}$ & $=$ & $\begin{array}{l}\text { resistances to flow of gel layer and } \\
\text { membrane, respectively }\end{array}$ & {$[\mathrm{MPa} \cdot \mathrm{s} / \mathrm{cm}]$} \\
\hline$r_{p}$ & $=$ & pore radius of gel layer & {$[\mathrm{cm}]$} \\
\hline$S_{D}, S_{F}$ & $=$ & $\begin{array}{l}\text { steric hindrance factors for diffusion } \\
\text { filtration flow, respectively }\end{array}$ & and \\
\hline$\Delta X$ & $=$ & thickness of gel layer & [cm] \\
\hline$\alpha$ & $=$ & coefficient of pressure dependency & {$[-]$} \\
\hline$\mu$ & $=$ & viscosity & {$[\mathrm{Pa} \cdot \mathrm{s}]$} \\
\hline$\sigma$ & $=$ & reflection coefficient & {$[-]$} \\
\hline \multicolumn{4}{|c|}{$\langle$ Subscripts $\rangle$} \\
\hline$g$ & & gel layer & \\
\hline 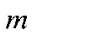 & $=$ & membrane & \\
\hline
\end{tabular}
of $\Delta X$ and $r_{p}$ as defined by Eqs. (13) and (14).
Nomenclature

\section{Literature Cited}

1) Bean, C. P.: "Membranes vol. 1: Macroscopic Systems and Models", G. Eisenman ed., p. 1 (1972).

2) Dorson, W. J., Jr., D. J. Cotter and V. B. Pizziconi: Trans. Amer. Soc. Artif. Int. Organs, 21, 132 (1975).

3) Hoshino, S. and K. Sato: Kagaku Kōgaku, 31, 961 (1967).

4) Jagur-Grodzinski, J. and O. Kedem: Desalination, 1, 327 (1966).

5) Kimura, S. and S. Nakao: ibid., 17, 267 (1975).

6) Nakao, S. and S. Kimura: ACS Symposium Series, No. 154, Synthetic Membranes: vol. 2, p. 119 (1981).

7) idem: J. Chem. Eng. Japan, 14, 32 (1981).

8) idem: ibid., 15, 200 (1982).

9) Nakao, S., T. Nomura and S. Kimura: AIChE J., 25, 615 (1979).

10) Nomura, T. and S. Kimura: Proc. Int. Congress on Desalination and Water Re-Use, Vol. 3, p. 57 (1979).

11) Sheppard, J. D., D. G. Thomas and K. C. Channabasappa: Desalination, 11, 385 (1972).

12) Shor, A. J., K. A. Kraus, W. T. Smith, Jr. and J. S. Johnson, Jr.: J. Phys. Chem., 72, 2200 (1968).

13) Verniory, A., R. Du Bois, P. Decoodt, J. P. Gassee and P. P. Lambert: J. Gen. Physiol., 64, 489 (1973). 